\title{
IMPROVING THE GAIN-FREQUENCY PERFORMANCE OF CASCADED SINGLE-STAGE DISTRIBUTED AMPLIFIERS WITH BANDPASS FILTER STRUCTURE
}

\author{
Metin Yazgi Ali Toker \\ e-mail: metin@ehb.itu.edu.tr alitoker@ehb.itu.edu.tr \\ Istanbul Technical University, Faculty of Electrical and Electronics Engineering, Department of Electronics and \\ Communication Eng., 80626, Maslak, Istanbul, Turkey
}

Key words: Distributed amplifiers, gain-frequency performance, bandpass structure

\begin{abstract}
In this paper a bandpass filter structure is proposed for realizing the artificial transmission lines of cascaded singlestage distributed amplifiers (CSSDA) and the basic analysis and design of the bandpass structured CSSDA (BP-CSSDA) is demonstrated. Some of the lower frequency region in the gain-frequency band of CSSDA is lost because of the bias components. Using the bandpass structure for realizing the artificial transmission lines, this lost frequency region can be added to the upper limit of the gain-frequency band. Therefore, BP-CSSDA gives more bandwidth than CSSDA using the lowpass structured lines. The gain can be increased by increasing the interstage termination impedance $\left(Z_{0 \text { int }}\right)$ without decreasing the bandwidth. A graphical method for determining the maximum $Z_{0 \text { int }}$ is introduced in this paper. The simulation results given in this paper show the advantage of using the BP structured artificial lines in CSSDA.
\end{abstract}

\section{INTRODUCTION}

The multioctave behaviour of conventional distributed amplifier (CDA) makes it one of the most investigated and realized broadband circuits [1-7]. But the gain performance of CDA is limited to about 7-14 dB. To overcome this disadvantage, in the literature, a novel broad-band amplifier has been presented [8-10]: Cascaded Single-Stage Distributed Amplifier (CSSDA). CSSDA has the same basic concept as CDA: the artificial transmission line. The gain of CSSDA is significantly higher than CDA using the same number of active devices. CSSDAs have three different artificial lines: input line, interstage line and output line. In the literature, all these lines have been analyzed and designed as lowpass structured lines [9-10]. On the other hand, when bias components are taken into account, it can be observed that these lines are similar to bandpass structured lines [7]. Since the bandpass structure proposed in this paper has necessary bias components, to obtain optimum gain-frequency performance the bandpass structure is more convenient for realizing the artificial lines of CSSDA.
Previous works made on CSSDA have been focused on the gain [9-10]. Those works have shown that the gain can be increased by increasing the interstage termination impedance $\left(Z_{0 \text { int }}\right)$ without decreasing the bandwidth. A graphical method obtained for simplified FET model can be used to determine the maximum $\mathrm{Z}_{0 \text { int }}$.

In this paper, the analysis and design of the proposed BP structured CSSDAs are given and the graphical method for the determination of the maximum $\mathrm{Z}_{0 \text { int }}$ is introduced. The simulation results given in this paper show the advantage of using the BP structured artificial lines in CSSDA.

\section{ANALYSIS OF THE BP-CSSDA}

A schematic representation of a CSSDA is shown in Figure-1. As explained above, CSSDA has three types of artificial transmission lines; the input line is made up of the input capacitance of the active device and added inductances, the interstage line is constructed by using the output capacitance of an active device and input capacitance of the next active device with added inductances, the output line is formed by the output capacitance of the last active device and added inductances. In general case, FET is used as active device.

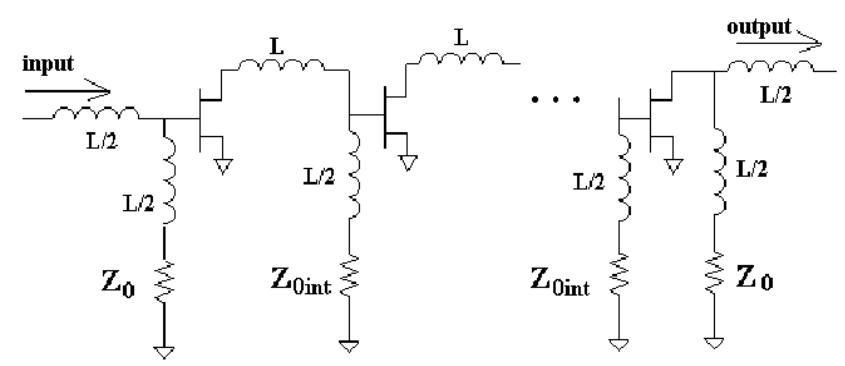

Figure-1 A schematic representation of a CSSDA

Shunt inductances for biasing the input and output ports of active devices and, serial capacitances for obtaining dcblock between the input and output ports must be used in the amplifier circuit. The artificial lines with the bias components are similar to a bandpass structure proposed 
in the literature for shifting the gain-frequency band of CDA [7]. Using the bandpass structure instead of lowpass structure is a more convenient way to obtain optimum gain-frequency performance of CSSDA, because the proposed BP structure has the necessary bias components. A schematic representation of a BP-CSSDA is shown in Figure-2.

Generally, the simplified FET model has been used for the basic analysis and design of DAs $[2,3,7,9,10]$. The simplified FET model is shown in Figure- 3 and the input, interstage and output lines of a BP-CSSDA are shown in Figure $-4,-5$ and -6 , respectively.

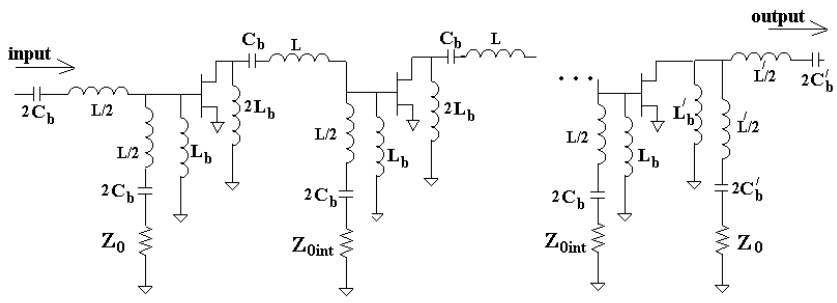

Figure-2 A schematic representation of the bandpass structured CSSDA.

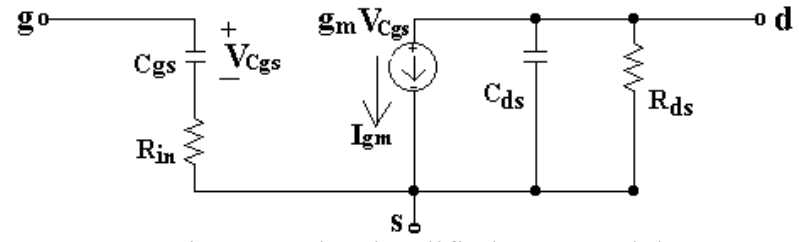

Figure-3 The simplified FET model.

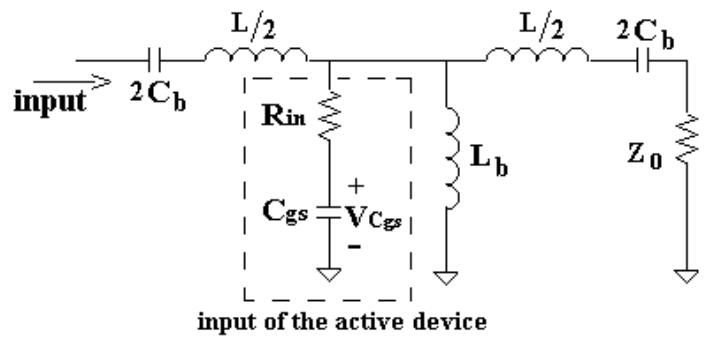

Figure.4 The input line of the BP-CSSDA.

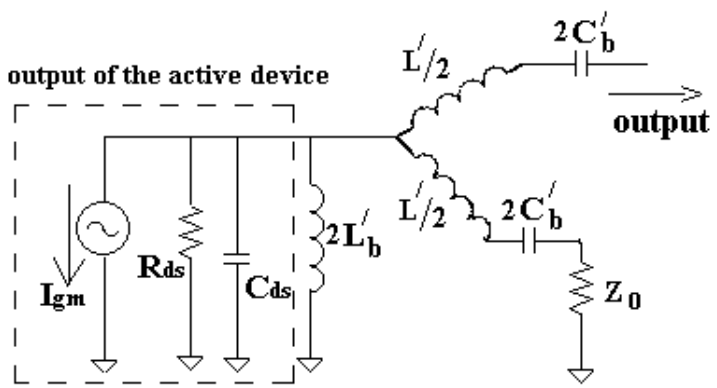

Figure- 6 The output line of the BP-CSSDA

The optimum performance of the BP lines [7] is obtained under the condition of

$$
\mathrm{LC}_{\mathrm{b}}=\mathrm{L}_{\mathrm{b}} \mathrm{C}_{\mathrm{gs}}
$$

For this case the characteristic impedance of the lines is

$\mathrm{Z}_{0}=\sqrt{\frac{\mathrm{L}}{\mathrm{C}_{\mathrm{gs}}}}=\sqrt{\frac{\mathrm{L}_{\mathrm{b}}}{\mathrm{C}_{\mathrm{b}}}}$

The geometric-center frequency of the band is

$\omega_{0}=\sqrt{\frac{1}{\mathrm{~L} \mathrm{C} \mathrm{C}_{\mathrm{b}}}}=\sqrt{\frac{1}{\mathrm{~L}_{\mathrm{b}} \mathrm{C}_{\mathrm{Cgs}}}}$

From Eq. (2)

$\frac{\mathrm{C}_{\mathrm{b}}}{\mathrm{Cgs}}=\frac{\mathrm{L}_{\mathrm{b}}}{\mathrm{L}}=\mathrm{k}$

It can be seen from the passive filter transformations [11] that the BP structure has the same bandwidth as that of the LP structure and the lower limit of the band in BP case is

$\mathrm{f}_{\mathrm{L}}=\left(\frac{1+\sqrt{1+\frac{1}{\mathrm{k}}}}{2}-1\right) \frac{1}{\pi \sqrt{\mathrm{LC}_{\mathrm{gs}}}}=\mathrm{bf}_{\mathrm{c}}$

In this equation, $\mathrm{f}_{\mathrm{c}}$ is the cut-off frequency of the LP line

$f_{c}=1 / \pi \sqrt{L_{\text {gs }}}$

In Eq.(5) $b$ gives the ratio of the lower limit in the BP case to the cut-off frequency in the LP case. $\mathrm{k}$ is found according to the aimed lower limit of the gain-frequency band. As a result, the band is shifted as $b_{c}$. 
In $\mathrm{BP}$ case, the gain at the geometric center frequency for the ideal lossless $\left(\mathrm{R}_{\mathrm{in}}=0, \mathrm{R}_{\mathrm{ds}}=\infty\right) \mathrm{N}$-cascaded single-stage distributed amplifier is given by

$\mathrm{S}_{21}=\frac{1}{2} \mathrm{~g}_{\mathrm{m}}^{\mathrm{N}} \mathrm{Z}_{0 \text { int }}^{(\mathrm{N}-1)} \mathrm{Z}_{0}$

In Eq.(7), $g_{m}$ is the transconductance of the active device and $\mathrm{Z}_{0}$ is the characteristic impedance of the input and output lines. Eq.(7) gives also the gain of LP-CSSDA for DC case [9].

For the simplified FET model, Eq. (7) changes because of $R_{d s}$ and $R_{i n}$. $R_{\text {in }}$ causes a shunt conductance at the input of the active device which increases with frequency [2]. The shunt conductance in the line caused by $\mathrm{R}_{\text {in }}$ for each transistor can be approximated as

$\mathrm{G} \cong \omega^{2} \mathrm{C}_{\mathrm{gs}}^{2} \mathrm{R}_{\text {in }}$

Taking the effects of $R_{d s}$ and $R_{\text {in }}$ into account, the gain is obtained as

$\mathrm{S}_{21}=\frac{1}{2} \mathrm{~g}_{\mathrm{m}}^{\mathrm{N}}\left(\mathrm{Z}_{0 \text { int }} / / \mathrm{R}_{\mathrm{ds}} / / \mathrm{G}^{-1}\right)^{\mathrm{N}-1}\left(\mathrm{Z}_{0} / / \mathrm{R}_{\mathrm{ds}}\right)$

\section{INCREASING THE TERMINATION IMPEDANCES}

As in the LP case, an increase in the value of the interstage termination impedances causes the gain to increase and the gain flatness to improve [9-10]. To obtain optimum performance, the values of the components which are serial to the termination impedance must be changed together with the value of the termination impedance. In the LP case, only serial inductance is changed, because there is no serial capacitance to the termination impedance. On the other hand, in the BP case, the values of the serial inductance and serial capacitance are changed into the convenient values. In the LP case, the value of the inductance is increased. This is valid for the BP case, but the value of the capacitance is decreased. The decrease in the value of the capacitance must be relatively the same as the increase in the value of the inductance.

Figure-7 shows the performance of the BP inter-stage lines for the lossless case. The increase of the inter-stage termination impedance causes the gain to increase in the middle of the band. The increase of the inductance improves the performance between the middle and upper regions of the band. And, the decrease of the capacitance improves the performance between the middle and lower regions of the band.

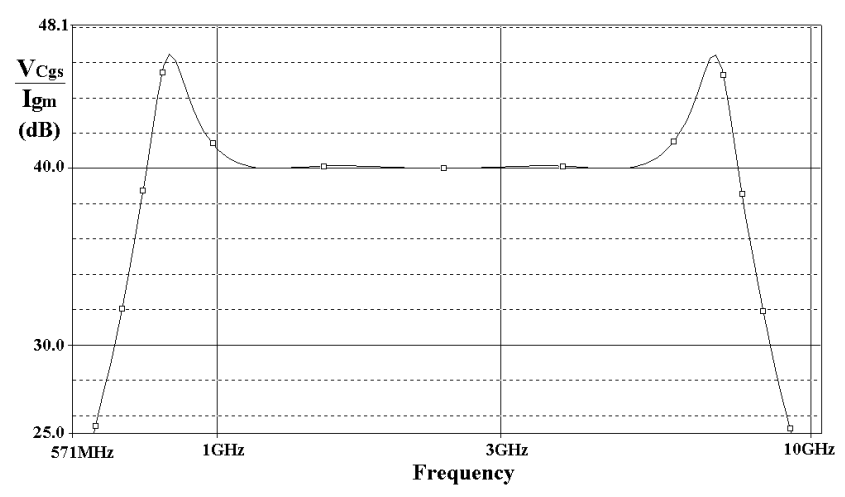

Figure-7 The performance of the BP inter-stage lines in the lossless case.

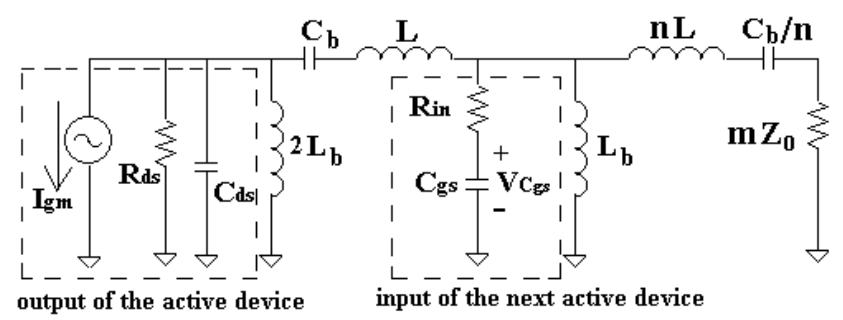

Figure- 8 The modified case of the interstage line.

The interstage line after increasing the termination impedance is shown in Figure-8. In Figure-5 the values of $\mathrm{n}$ and $\mathrm{m}$ are $1 / 2$ and 1 , respectively. In the modified case their values are increased. Analyzing the circuit in Figure8 for $\mathrm{V}_{\mathrm{Cgs}} / \mathrm{I}_{\mathrm{gm}}$, we obtain a second-order equation of

$\mathrm{C}_{2} \mathrm{n}^{2}+\mathrm{C}_{1} \mathrm{n}+\mathrm{C}_{0}=0$

The coefficients $\mathrm{C}_{0}, \mathrm{C}_{1}$ and $\mathrm{C}_{2}$ depend on values of $\mathrm{k}, \mathrm{Z}_{0}$, $R_{i n}, R_{d s}, m$, the ratio (p) of the gain at any frequency to the gain at the center frequency and the normalized value (a) of the frequency to the parameters of the lines. a is

$\mathrm{a}=\omega_{\mathrm{p}} \sqrt{\mathrm{LC}_{\mathrm{gs}}}$

Using Eq.(10), the necessary value of $n$ can be obtained for any value of $\mathrm{m}$. This idea has been realized by means of a MATLAB program. In this program, a is swept and $n$ is calculated by means of Eq.(10) for given values of $p, k$, $\mathrm{Z}_{0}, \mathrm{R}_{\mathrm{in}}, \mathrm{R}_{\mathrm{ds}}$ and $\mathrm{m}$. If $\mathrm{n}$ is real for acceptable values of $\mathrm{a}$ (here, acceptable value means convenient bandwidth), the values of $n$ and $m$ are convenient for the design. The higher values of $\mathrm{n}$ and $\mathrm{m}$, the better gain-frequency performance.

Tablo-1 The simplified FET model parameters of NE72089A

\begin{tabular}{|l|l|}
\hline $\mathrm{C}_{\mathrm{gs}}$ & $0.94 \mathrm{pF}$ \\
\hline $\mathrm{R}_{\mathrm{in}}$ & $10 \Omega$ \\
\hline $\mathrm{g}_{\mathrm{m}}$ & $65 \mathrm{mS}$ \\
\hline $\mathrm{C}_{\mathrm{ds}}$ & $.43 \mathrm{pF}$ \\
\hline $\mathrm{R}_{\mathrm{ds}}$ & $160 \Omega$ \\
\hline
\end{tabular}




\section{A DESIGN EXAMPLE AND SIMULATION RESULTS}

The simplified FET model parameters of the transistor NE72089A (from NEC) is given in Table-1. An important point of the design is the value of the interstage termination impedance. The higher value of the impedance, the higher gain. But, there is a top limit for its value because of the gain-flatness. Figure- 9 and 10 show the real part and imaginal part curves of $n$. The curves have been obtained for chosen values of $m, p, k$ and $Z_{0}$. The values are given in Table-2. For $\mathrm{k}=2, \mathrm{~b}$ is calculated as 0,11 by using Eq.(5). This is a convenient value for the general case and means that the gain-frequency band is shifted approximately $\% 11$ into a higher frequency region according to the lowpass case. An increase in the value of $\mathrm{k}$ increases the shift of the gain-frequency band. $\mathrm{p}$ is chosen according to the number of the stages and determines the gain-flatness. $3 \mathrm{~dB}$ is an acceptable value for the maximum ripple in the band. Taking the negative and positive values of the ripples into account, $p$ is determined as $\pm 1,5 \mathrm{~dB} /$ (the number of the stages -1 ).

In general case, it is not convenient that the characteristic impedance of the interstage line is made higher than that of the input line $\left(\mathrm{Z}_{0}=50 \mathrm{Ohm}\right)$. Because any increase in the characteristic impedance of the artificial lines means a decrease in the bandwidth of the lines $[1,3,6]$.

Tablo-2 The values of $\mathrm{m}, \mathrm{p}, \mathrm{k}$ and $\mathrm{Z}_{0}$ used to obtain the value of $n$.

\begin{tabular}{|l|l|}
\hline$Z_{0}$ & $50 \mathrm{Ohm}$ \\
\hline $\mathrm{K}$ & 2 \\
\hline $\mathrm{M}$ & 1.6 \\
\hline $\mathrm{P}$ & \pm 0.5 \\
\hline
\end{tabular}

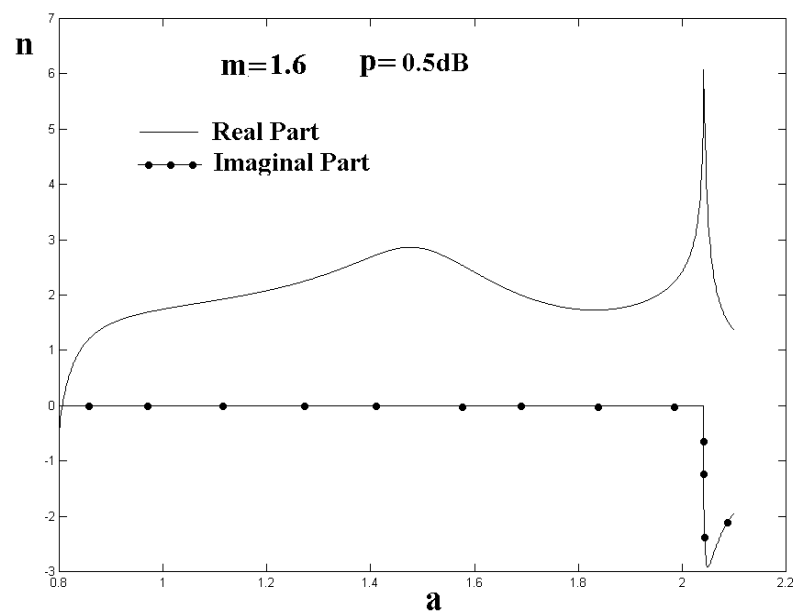

Figure-9 The real part and imaginal part curves of $n$ for $\mathrm{p}=0,5 \mathrm{~dB}$.

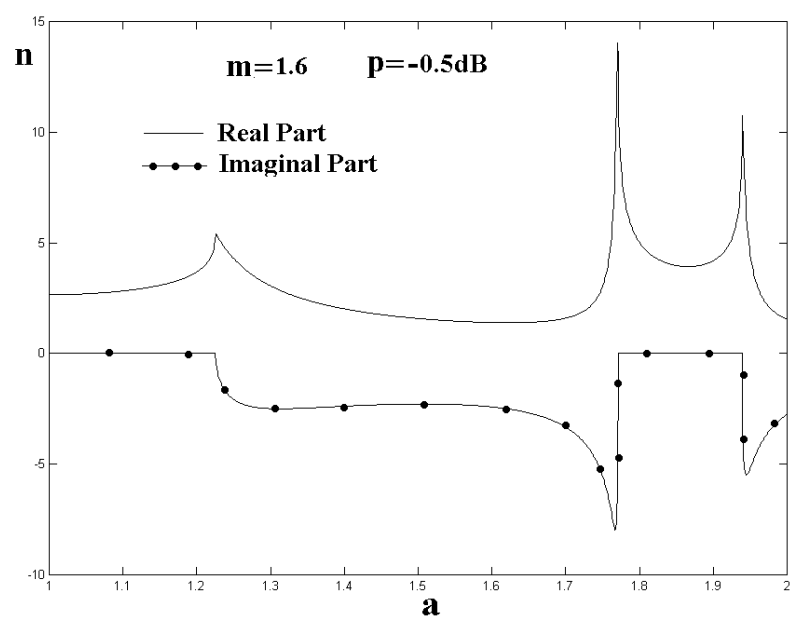

Figure-10 The real part and imaginal part curves of $n$ for $\mathrm{p}=-0,5 \mathrm{~dB}$.

Figure- 9 has been obtained for $\mathrm{p}=0.5 \mathrm{~dB}$. It is clear that the real values of $n$ can be used. Observing the regions in which the imaginal part of $n$ is zero, the value of $n$ is determined. This condition is valid in most of the imaginal part curve in Figure-9. It is seen from this figure that the most convenient value of $\mathrm{n}$ is approximately 6 for $p=0.5 \mathrm{~dB}$. Figure- 10 has been obtained for $\mathrm{p}=-0.5 \mathrm{~dB}$. The imaginal part is not zero in most of the curve in Figure10. In this figure, the most convenient value of $\mathrm{n}$ can be found by investigating two regions: one between $a \cong 1.78$ and $a \cong 1.94$ and the other $a \leq 1,25$. It can be seen from these regions that the values of $\mathrm{n}$ higher than 5 are obtained only in the region between $\mathrm{a} \cong 1.78$ and $\mathrm{a} \cong 1.94$. All these observations show that $\mathrm{n}=6$ is given by both the characteristics as a convenient value. As a result, $\mathrm{m}=1.6$ and $\mathrm{n}=6$ are obtained as convenient values. Actually, these values give only a good starting point for the design. The values of $n$ and $m$ must be changed for the optimum performance in the next steps of the design.

The performance of the BP-CSSDA is investigated by means of the simulations made by using MicrowaveHarmonica. To obtain a comparison between LP and BP CSSDA, simulations are made on both amplifiers using NE72089A as active device. The number of the active devices is four. S parameters of the transistor have been used in the simulations.

$\mathrm{S}_{21}$ has been maximized for the both amplifiers by taking the values of $S_{11}$ and $S_{22}$ into account. Effects of the interstage lines on $S_{11}$ and $S_{22}$ are very little. Therefore, the inter-stage lines are very flexible for the optimization of $S_{21}$. The input and output lines determine $S_{11}$ and $S_{22}$, respectively. But, this does not mean that any change on the input and output lines must not be made. 
At the end of the optimization, $\mathrm{n} \cong 5$ and $\mathrm{m} \cong 1.8$ have been obtained for the BP-CSSDA. These values are very near to the values given by the graphical method. At the same time, the input line and output line parameters have been changed for the optimization. The change of the input line parameters is not very much. Because the bandwidth of the input line is equal to that of the amplifier. On the other hand, the output line has more bandwidth than that of CSSDA. Therefore, the output line parameters are more flexible than the input line parameters.

For the LP-CSSDA, the same way has been used to obtain optimum gain-frequency performance. The inductances which are necessary to bias the drain and gate ports of the active devices have been added to the circuit. The values of the inductances have been chosen $30 \mathrm{nH}$ to obtain the same lower frequency limit as the BP-CSSDA. On the other hand, the highest value for the inductances used in the BP-CSSDA is $12 \mathrm{nH}$.

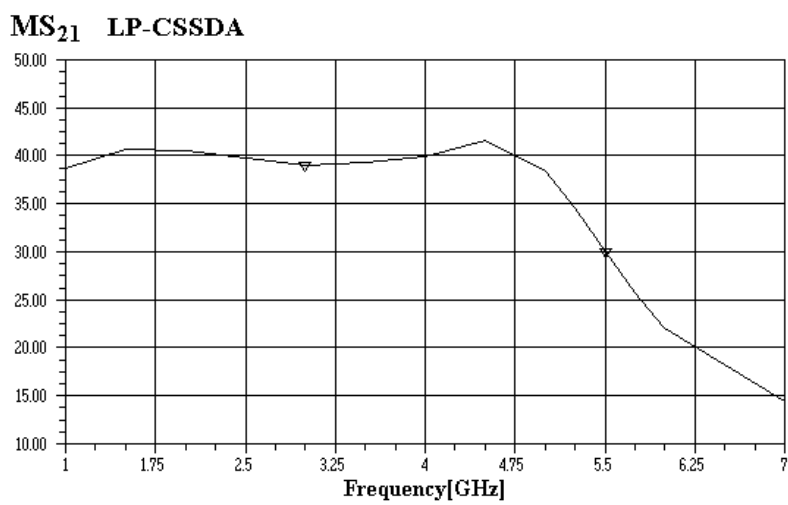

Figure-11 $\mathrm{S}_{21}$ performance of the LP-CSSDA.

\section{MS $_{21}$ BP-CSSDA}

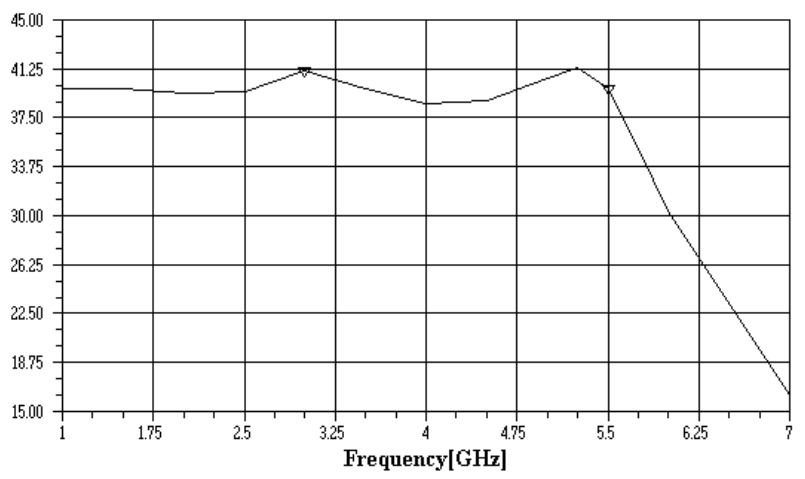

Figure-12 $\mathrm{S}_{21}$ performance of the BP-CSSDA.

The simulation result given in Figure-11 for the LPCSSDA shows that the average value of $S_{21}$ is $40 \mathrm{~dB}$ over $1-5 \mathrm{GHz}$. It is clear from Figure-12 that the average value of $\mathrm{S}_{21}$ for the BP-CSSDA is $40 \mathrm{~dB}$ over $1-5,5 \mathrm{GHz}$. The maximum ripple for both amplifiers is $3 \mathrm{~dB}$ in the gainfrequency band. As a result, the BP-CSSDA has a bandwidth approximately $12 \%$ more than that of the LP CSSDA. The simulation results clearly show the advantage of using the BP structure for realizing the lines.

\section{CONCLUSION}

In this paper a bandpass filter structure has been proposed for realizing the artificial transmission lines of CSSDA and the basic analysis and design of the bandpass structured CSSDA has been demonstrated. Some of the lower frequency region in the gain-frequency band of CSSDA is lost because of the bias components. The simulation results have shown that this lost frequency region can be added to the upper limit of the gainfrequency band by using the BP structure for realizing the artificial lines of CSSDA. The gain can be increased by increasing the interstage termination impedance $\left(\mathrm{Z}_{0 \text { int }}\right)$ without decreasing the bandwidth. In this paper a graphical method for determining the maximum value of the interstage termination impedance has been introduced.

\section{REFERENCES}

1. W. S. Percival, British Patent Specification No.460, 562, 1936

2. Y. Ayasli, S. W. Miller, R. Mozzi and L.K. Hannes, "Capacitively Coupled Travelling-Wave Power Amplifier", IEEE Trans. Microwave Theory Tech., vol. MTT-32, Dec. 1984, pp. 1704-1709

3. J. B. Beyer, S. N. Prasad and R. C. Becker, "MESFET distributed amplifier guidelines", IEEE Trans. Microwave Theory Tech., vol. MTT-32, pp. 268-275, March 1984

4. C. S. Aitchison, "The Intrinsic Noise Figure of the MESFET Distributed Amplifier", IEEE Trans. Microwave Theory Tech., vol. MTT-31, pp. 460-466, June 1985

5. A. Toker, Bipolar Tranzistorlu Dagilmis Parametreli Kuvvetlendiricilerde Yeni Olanaklar, PhD. Thesis, Institute of Science and Technology, I.T.U, 1986

6. T. T. Y. Wong. Fundamentals of Distributed Amplifiers, Artech House, Boston, 1993.

7. M. Yazgi, A. Toker, D. Leblebici, On Distributed Amplifiers with Bandpass Filter Structure, Proceedings of ELECO'99, Bursa, Turkey, pp. 128131, Dec. 1999.

8. B. Y. Banyamin, C. S. Aitchison and J. Y. Laing, "A New High Gain-broadband Amplifier Using Cascaded Single Stage Distributed Amplifiers", AsiaPasific Microwave Conference Proceedings, pp. 753756, 1998.

9. Ben Y. Banyamin, Michael Berwick, "Analysis of the Performance of Four-Cascaded Single Stage Distributed Amplifiers", IEEE Trans. Microwave Theory Tech., Vol. 48, No 12, pp. 2657-2663, Dec. 2000.

10. Avtar S. Virdee, Bal S. Virdee, "A Boradband 2 to 18 $\mathrm{GHz}$ Cascaded Reactively Terminated Single-Stage Distributed Amplifier, Microwave Journal, pp. 22-42, September 2000.

11. K. L. Su, Analog Filters, Chapman \& Hall, London, 1996 
